\title{
Governança Corporativa e Velocidade de Incorporação de Informações: Lead-Lag entre o IGC e o IBrX
}

(Corporate Governance and Information Incorporation Speed: Lead-Lag between the IGC and IBrX)

\author{
José Carneiro da Cunha Oliveira Neto* \\ Otávio Ribeiro de Medeiros** \\ Thiago Bergmann de Queiroz***
}

\section{Resumo}

Com base em dados intradiários em frequência de 15 minutos, o presente estudo investiga a relação entre o mercado de Governança Corporativa Diferenciada (IGC) e o Mercado Tradicional (IBrX). A hipótese testada é de que um melhor nível de Governança Corporativa reduz os custos associados à incorporação de novas informações aos preços dos ativos da empresa. Assim, empresas com melhor nível de governança teriam maior velocidade de incorporação de informações. A relação de cointegração entre as séries foi testada com o uso do método Engle-Granger, em dois estágios. O Vetor de Correção de Erros (VECM) e o teste de causalidade de Granger não permitem a rejeição da hipótese de maior velocidade de incorporação de informações do Mercado de Governança Diferenciada. Para estimativa do VECM, utilizou-se um modelo GARCH bivariado BEKK. Os resultados sugerem que o IGC encontra seu preço de equilíbrio mais rapidamente e que o IBrX converge para a relação de equilíbrio apontada pelo IGC.

Palavras-chave: governança corporativa; hipótese de mercados eficientes; GARCHBEKK; lead-lag.

JEL codes: G3; G39.

\section{Abstract}

Based on intraday data with a frequency of 15 minutes, the present study investigates the relationship between the high corporate governance market (IGC) and the traditional market (IBrX). The hypothesis tested is that a higher level of corporate governance reduces the cost associated to incorporating new information to asset prices, and so firms with higher governance incorporate information faster. The co-integration relationship between the time series was tested using the Engle-Granger method in two stages. The vector error correction

Submetido em 16 de outubro de 2010. Reformulado em 3 de agosto de 2011. Aceito em 4 de outubro de 2011. Publicado on-line em 15 de abril de 2012. O artigo foi avaliado segundo o processo de duplo anonimato além de ser avaliado pelo editor. Editor responsável: Márcio Laurini. O primeiro autor agradece ao apoio financeiro da Fundação de Amparo à Pesquisa de São Paulo (FAPESP). Reprodução parcial ou total e trabalhos derivativos permitidos com a citação apropriada da fonte.

*PPGA/Universidade de Brasília, Brasília, DF, Brasil. E-mail: joseccon@gmail.com

**PPGA/Universidade de Brasília, Brasília, DF, Brasil. E-mail: otavio@unb.br

***PPGA/Universidade de Brasília, Brasília, DF, Brasil. E-mail: thiago.bergmann@ hotmail.com 
model (VECM) and the Granger causality test do not permit the rejection of the hypothesis of faster incorporation of information for the high governance market prices. To estimate the VECM we used a bivariate GARCH BEKK model. The results suggest that the IGC finds its equilibrium price more rapidly and that the $\mathrm{IBrX}$ converges to the equilibrium relationship determined by the IGC.

Keywords: corporate governance; efficient markets hypothesis; GARCH-BEKK; lead-lag.

\section{Introdução}

O presente artigo tem como objetivo testar a hipótese de que um melhor nível de governança corporativa reduz os custos associados à incorporação de novas informações aos preços dos ativos da empresas. Dessa forma, é possível que a velocidade de incorporação de informações associada a ativos de empresas com níveis mais elevados de governança seja superior à de companhias com estruturas de governança mais fracas, fazendo com que os preços das ações das primeiras liderem os preços das ações das segundas, caracterizando um efeito Lead-Lag. Há estudos que tratam da relação entre qualidade da informação, transparência e redução dos custos de processamento de novas informações, mas nenhum trabalho sobre o impacto da governança corporativa na velocidade de incorporação de informações aos ativos e consequentes efeitos Lead-Lag foi encontrado pelos autores.

Para que haja possibilidade de maior velocidade de incorporação de informações, os compromissos de transparência e respeito contratualmente formalizados pelas companhias têm de ser críveis no mercado, o que ocorre através de um processo de construção de boa reputação do próprio mercado de governança diferenciada perante a contraparte: o investidor.

A velocidade de incorporação de informações do mercado de governança diferenciada será maior quanto melhor for a reputação da estrutura contratual privada que a sustenta. Quanto pior for a legislação corporativa e o sistema jurídico de determinado país e mais eficazes forem os instrumentos contratuais e as instituições que fundamentam o mercado de governança diferenciada, maior será a diferença na velocidade de incorporação de informações entre os mercados; maior será, então, o efeito liderança. Nesse sentido, o efeito Lead-Lag pesquisado não está relacionado apenas a uma eventual liderança entre mercados, mas, também, à avaliação de qual mercado encontra primeiro o equilíbrio após um choque e qual converge posteriormente para esse equilíbrio.

A estratégia de estimação do efeito adotada é uma variação da utilizada por Brooks et al. (2001) e Pati \& Rajib (2010).

Ela tem como principal objetivo analisar a convergência das séries à relação de equilíbrio de longo prazo.

O estudo está focado na estimação do efeito Lead-Lag, com base na análise da velocidade de incorporação de informações entre o mercado de governança diferenciada e o mercado convencional. Não foram realizados testes empíricos para relação entre preços e fundamentos. Apesar do uso da teoria neoclássica 
permitir a extrapolação de algumas conclusões, essas devem ser observadas com cautela.

A análise do efeito Lead-Lag começa em maio de 2009 e termina em fevereiro de 2010, período no qual o mercado brasileiro de governança diferenciada já pode ser considerado razoavelmente consolidado. Os dados são intradiários na frequência de 15 minutos, sendo utilizada a cotação de fechamento. Perfazem 5.582 observações de preços e 5.581 observações de retornos. A hipótese é de que os instrumentos contratuais privados que fundamentam o mercado de governança diferenciada aumentam à velocidade de incorporação de informações aos ativos desse segmento, quando comparado com o mercado tradicional. Dessa maneira, deveria existir um efeito Lead-Lag entre o Índice de Governança Diferenciada (IGC) e o Índice Brasil de Ações (IBrX). Os dados utilizados foram obtidos no sistema CMA de cotação de ativos.

Para estimativa do efeito liderança, com foco direcionado ao estudo de qual série atinge primeiro o equilíbrio de longo prazo, estimou-se um modelo VECMGARCH-BEKK; adicionalmente foi testada a causalidade de Granger entre o IBrX e o IGC. A evidência do efeito liderança é diagnosticada ou refutada pela significância estatística das variáveis defasadas. Já a avaliação de qual série atinge primeiramente o equilíbrio e qual converge posteriormente para essa posição foi realizada por meio do sinal e da significância estatística do parâmetro estimado para o Mecanismo de Correção de Erro (ECM). A interpretação do coeficiente segue Brooks et al. (2001).

As evidências encontradas não permitem a rejeição da hipótese proposta e indicam que o mercado de governança lidera o mercado tradicional. Foi encontrada causalidade unidirecional de Granger do IGC para o IBrX, assim como indícios, apontados pelo sinal e significância estatística do Mecanismo de Correção de Erros (ECM), de que o mercado de governança diferenciada encontra seu preço de equilíbrio de longo prazo mais rapidamente que o mercado tradicional, e que o último converge para a relação de equilíbrio apontada pelo primeiro.

Os resultados encontrados têm implicações positivas para o atual movimento de melhoria das práticas de governança nas empresas brasileiras de capital aberto, pois referendam os esforços feitos pela BM\&F Bovespa, assim como pelos órgãos regulatórios e demais participantes do mercado, para o aperfeiçoamento do mercado de capitais brasileiro. Embora o presente artigo não tenha a pretensão nem a possibilidade de demonstrar categoricamente, é possível que a redução dos custos de processamento da informação esteja associada à redução de problemas de assimetria de informação e à percepção pelo mercado de melhorias na qualidade das informações prestadas e do ambiente legal.

\section{Referencial Teórico}

A informação possui papel central na Teoria Financeira. Entre diferentes correntes de pensamento, tais como a abordagem neoclássica e finanças comportamentais, existem divergências sobre como ela é incorporada ao preço dos ativos, 
ou na forma como os indivíduos a transformam em ação efetiva, mas todas concordam com sua importância.

Usualmente, os estudos sobre a dinâmica da incorporação de informações ao preço, à estrutura e ao design do mercado, que inclui a relação entre formas contratuais e formação de preços, e a habilidade dos participantes em observar informações relevantes ao processo de negociações são concentrados em uma subárea científica conhecida como Microestrutura do Mercado (Madhavan, 2000). Não só mercados de capitais em vários países possuem microestruturas diferentes, como o mesmo mercado de capitais pode ter consideráveis distinções com relação à transparência e à estrutura contratual entre as diversas classes de ativos nele negociadas.

Fama (1991) argumenta que para qualquer discussão sobre equilíbrio é necessário que se leve em conta a maneira como a informação é incorporada ao preço. Lucas $(1978,1986)$ defende que o processo de aprendizagem e os demais incentivos existentes na economia são suficientes para que os indivíduos façam o melhor uso possível da informação disponível. Por sua vez, Byrne \& Brooks (2008) acreditam que nem sempre os investidores processam as informações disponíveis de maneira correta ou, quando o fazem, podem, ainda assim, assumir comportamentos questionáveis.

A qualidade e a complexidade da informação são fundamentais para seu uso efetivo e têm consequências diretas sobre o custo de capital e o valor de mercado de uma empresa, assim como sobre a velocidade de incorporação de informações pelo mercado. Easley \& O'Hara (2004) encontraram evidências de que empresas que estejam em mercados com grandes níveis de informação privada possuem maior custo de capital, que cai na medida em que a informação pública se torna dominante. Botosan et al. (2004) apontam para uma relação inversa entre a precisão das informações públicas e o custo de capital, enquanto, na medida em que a precisão das informações privadas aumenta, o custo de capital da empresa também aumenta. Em ambos os casos, quanto maior a assimetria de informação entre os agentes econômicos, maior o custo de capital das empresas.

Para Bushman et al. (2004), uma gestão pouco transparente dificulta o processo de acompanhamento da empresa, o que aumenta a demanda por melhores níveis de governança, que, em última instância, deve melhorar o nível de transparência.

Plumlee (2003) analisou o impacto da complexidade da informação em seu uso prático. Segundo a autora, na medida em que a informação ganha complexidade torna-se mais difícil sua incorporação nas estratégias adotadas pelos investidores; em casos extremos, onde a informação é exageradamente complexa, ela não é sequer processada. Essa conclusão segue Jensen (1978), para quem uma informação só é utilizada quando seu benefício marginal supera seu custo de processamento. Nessa linha, há forte coerência com o apontado por Easley \& O'Hara (2004) e Botosan et al. (2004): na presença de dificuldades para processar informação, o custo do capital cresce para compensar o risco adicional. 
Ainda em Plumlee (2003), a autora evidencia que informações mais complexas demandam mais tempo para serem incorporadas. Diferenças entre tempo de incorporação de informação também são encontradas por Brenann et al. (1993), onde firmas maiores, acompanhadas por maior número de analistas, incorporam novas informações a seus preços de mercado de maneira mais rápida do que empresas menores, acompanhadas por menos investidores.

Sunders (1992) questiona a idéia de que, em um equilíbrio competitivo, os ativos financeiros revelam a informação de maneira que o fluxo de caixa descontado de se produzir a informação é zero. Para o autor, quando o custo da informação é fixado em um patamar suficientemente alto, agentes informados e desinformados se distribuem no mercado de tal forma que os informados conseguem recuperar seus investimentos.

Oliveira \& Medeiros (2009) procuraram evidenciar o efeito Lead-Lag entre os mercados acionários norte-americano e brasileiro, o que propiciaria previsibilidade aos preços das ações negociadas no Brasil, indicando uma segmentação entre esses mercados e trazendo possibilidade de arbitragem. Embora os autores tenham constatado que o Ibovespa é, em grande parte, explicado pelo movimento antecedente do Índice Dow Jones, os resultados mostraram que a realização de arbitragem não é economicamente viável em decorrência dos custos de transação, não contrariando assim a Hipótese do Mercado Eficiente.

Num estudo envolvendo ações de um mesmo mercado acionário, o mercado indiano, Poshakwale \& Theobald (2004) constataram efeitos Lead-Lag entre ações de empresas com alta capitalização e ações de empresas de baixa capitalização, onde a liderança é exercida pelas primeiras. Os autores atribuem tais efeitos a uma maior velocidade de incorporação de informações pelas ações de alta capitalização, em função de uma maior cobertura pelos analistas, disponibilidade de informações, dentre outros fatores.

A relevância da informação para a explicação do efeito Lead-Lag pode ser diretamente derivada da colocação de Fama (1991) sobre a necessidade de se especificar corretamente o processo pelo qual a informação é absorvida pelos ativos, em um modelo de equilíbrio geral.

De qualquer forma, há que se levar em consideração que, embora a visão neoclássica de finanças assuma que a relação de equilíbrio reflete os fundamentos das empresas, há razões teóricas e também evidências empíricas que questionam essa suposição no mercado acionário, de modo que os resultados obtidos no presente trabalho devem ser vistos com cautela, uma vez que as diferenças na velocidade de incorporação de informação entre os dois mercados em pauta poderiam ser explicadas por outros fatores (Cutler et al., 1989, Sunders, 1992, Brenann et al., 1993, Chan, 2003). 


\section{Governança Corporativa}

A Governança Corporativa trata dos mecanismos através dos quais os fornecedores de capital para uma empresa têm garantias que receberão os retornos de seus investimentos. Tais mecanismos podem ser definidos como instituições econômicas e legais passíveis de alteração pelo processo político (Shleifer \& Vishny, 1997). Empresas com bons níveis de governança atuam segundo regras e procedimentos claros, com elevados níveis de transparência quanto à divulgação de informações.

Para Carvalho (2002), a Governança Corporativa representa os princípios que governam o processo de decisório dentro de uma empresa, com o objetivo central de resolver, ou garantir que não ocorram, problemas de agência.

As relações de agência são, segundo Jensen \& Meckling (1976), contratos onde uma ou mais pessoas, definidas como principal, delegam a terceiro (agente) a autoridade para tomar decisões que afetam seu bem-estar. "Se ambas as partes da relação são maximizadoras de utilidade, há boas razões para se acreditar que o agente não agirá sempre no melhor interesse do principal" (Jensen \& Meckling, 1976, p. 308).

Roe (2005) argumenta que os problemas centrais da Governança Corporativa podem ser divididos em três grupos distintos; são eles:

- Governança Corporativa Vertical - custos de agências gerenciais: trata dos problemas relacionados à garantia de que os gerentes, em empresas de capital aberto com propriedade dispersa, terão como objetivo central a maximização do bem-estar de seus acionistas;

- Governança Corporativa Horizontal - limitação da ação de acionista majoritário: enquanto, no universo anglo-saxão, a governança corporativa vertical é o principal problema em empresas abertas, no restante do mundo, companhias de capital aberto não são caracterizadas pela propriedade dispersa, mas sim pela presença de um acionista, ou pequeno grupo de acionistas, que controla a empresa. Nessa situação, o principal risco enfrentado pelos demais acionistas não é a expropriação por parte de administração, mas sim pelo acionista que detém o controle acionário da firma;

- Governança Corporativa Externa - legitimidade corporativa: esta dimensão trata da legitimidade da governança da firma para a sociedade e da possibilidade de incursões externas nas decisões da companhia. Roe (2005) apresenta uma sinterização abstrata dessa idéia: "Como a normatização pode organizar um negócio que sobrevive política e socialmente?" e "Como as instituições corporativas podem ser positivamente adaptadas para serem socialmente legítimas?" (Roe, 2005, p. 376). Duas abordagens são possíveis:

- defensiva: agentes internos estruturam instituições para protegê-los de incursões externas; e, 
- legitimidade determinada por externos: políticas restringem a maneira como a firma pode ser governada, com o objetivo de permitir a implementação de determinadas políticas públicas, como relações trabalhistas específicas.

LaPorta et al. $(1998,2000)$ listam evidências de que diferentes estruturas legais afetam diretamente a estrutura de propriedade da empresa. Em países com estruturas legais de proteção dos acionistas eficientes, as companhias de capital aberto tendem a ter propriedade difusa e o mercado financeiro melhor desenvolvido. Por outro lado, quando as estruturas que protegem o acionista/credores são limitadas, o mercado financeiro é menos desenvolvido e as firmas de capital aberto têm estrutura acionária concentrada. As diferentes regras corporativas vigentes em diversos países condicionam diretamente a forma como empresas se organizam. A fraca proteção legal do investidor aumenta o risco de expropriação por parte da administração, o que estimula a concentração da propriedade como forma de se resolver o problema. Por sua vez, a concentração aumenta a possibilidade de expropriação dos acionistas minoritários por parte dos majoritários, risco que pode depender de regras exógenas à empresa para ser mitigado.

Goergen \& Renneboog (2008) argumentam que companhias podem se desviar dos padrões nacionais de governança através de dispositivos contratuais. Nesse sentido, podem optar, a depender das restrições e incentivos que enfrentam, por aumentar ou reduzir a proteção ofertada aos acionistas. No Brasil, empresas de capital aberto podem manifestar contratualmente a adesão a níveis de governança corporativa diferenciada, sendo o Novo Mercado o ambiente de negociação na BM\&F Bovespa com o nível mais exigente, onde a companhia deve, inclusive, assinar, segundo Srour (2005), um termo onde elege uma câmara arbitral como local para discussão de eventuais litígios envolvendo os interessados nos ativos da companhia. Tal termo tem papel fundamental na garantia de que os compromissos assumidos pela empresa no tocante à governança sejam críveis, pois elimina a possibilidade da firma recorrer a subterfúgios jurídicos para impedir a punição por eventuais desvios.

Um impacto bem documentado de melhores práticas de governança diz respeito ao valor da firma. Gompers et al. (2003) encontraram, para o mercado americano, evidências de que firmas com bons níveis de governança valem mais. Silva (2004) e Silva \& Leal (2005) e Silveira et al. (2006) encontraram resultados semelhantes para o mercado de capitais brasileiro, Black (2001) para a Rússia e Joh (2003) para a Coréia. Bhojraj \& Sengupta (2003) defendem que melhores níveis de governança, ao reduzirem a assimetria de informação, afetam a rentabilidade e a classificação de risco de bônus corporativos e apontam forte relação entre a qualidade da governança e a classificação de risco do título. Por sua vez, Silveira et al. (2008) apontam que melhores níveis de governança são positivamente relacionados com a alavancagem financeira, sendo, provavelmente, determinantes da estrutura de capital. Tal conclusão segue Jensen \& Meckling (1976). 
Segundo LaPorta et al. (2000), o arcabouço legal é a fonte explicativa primária da forma como firmas se organizam, assim como principal determinante da probabilidade de expropriação do investidor. Para Williamson (1988), a oferta de bons serviços e bons níveis de governança deve ser simultaneamente examinada. Nesse sentido, defende que a qualidade da administração financeira de uma companhia está diretamente ligada à qualidade de sua governança. Black et al. (2009) evidenciam que as empresas brasileiras são bastante heterogênicas quanto à qualidade de sua governança.

Williamson (1983, 1984) e Romano (1996) acreditam que melhores práticas de governança podem ser benéficas para a empresa, mas que, do ponto de vista do investidor, as regras voluntariamente aceitas pelas companhias, ou propostas por mecanismos de auto-regulação, substituem apenas de forma imperfeita uma boa e eficiente norma legal.

Existem evidências de que boas práticas de governança afetam o valor e o risco de companhias de capital aberto (Silva, 2004; Silva \& Leal, 2005; Silveira, Barros e Famá, 2006). Também há evidência de que uma boa legislação corporativa tem reflexos positivos no valor de mercado, risco e estrutura de capital das firmas (LaPorta et al., 1998, 2000, Romano, 1996, Shleifer \& Vishny, 1997). Porém, pouco estudo foi dedicado ao impacto da legislação corporativa e da estrutura de governança na velocidade da incorporação de novas informações ao preço de mercado de ativos das empresas ou governos.

Melhores práticas de governança e legislação, assim como delimitação clara do campo de intervenção e atuação do governo, podem ter efeito direto sobre o grau de complexidade dos impactos de uma nova informação sobre o valor de mercado de uma empresa ou título. Afetam, assim, a velocidade com a qual a nova informação é incorporada pelo ativo.

Como impactos, pode-se interpretar as ações e comportamentos assumidos pelos agentes econômicos em face da nova informação. Em última análise, são essas ações que determinam as alterações no valor de mercado e no risco dos títulos e empresas.

A Governança Corporativa, já definida como mecanismos que garantam ao fornecedor de capital o retorno pelo investimento realizado, também pode ser caracterizada como uma relação contratual entre a empresa e seus fornecedores de capital. Essa relação é regida por uma série de instituições endógenas, como o estatuto social, e exógenas, como a legislação corporativa, à firma. Relações contratuais endógenas resolvem apenas parcialmente normas exógenas ruins; além disso, deve haver forte relação entre a qualidade da governança adotada pela companhia e a eficiência com a qual a administração financeira é executada (Williamson, 1983, 1984, 1988).

Empresas com melhores níveis de governança têm regras mais claras e transparentes de decisão, oferecem melhores estruturas de proteção aos fornecedores de capital e, ao menos em teoria, criam normas e aderem a regras que tornam tais promessas em compromissos críveis. 
Há, portanto, algum tipo de estrutura que garante ou, ao menos, aumenta drasticamente a probabilidade do cumprimento, por parte da companhia, das promessas realizadas. Fazer com que fornecedores de capital acreditem em tais promessas não é, necessariamente, tarefa fácil. Para isso, firmas contam com dois instrumentos básicos: adesão a normas via contratos que imponham severas penas aos desvios; e construção de reputação.

Quando mais eficientes forem as instituição que garantem o cumprimento dos contratos em determinado país, menos necessária será a construção de reputação. A adesão a determinado tipo de relação contratual com os fornecedores de capital, que em caso de desvio será facilmente imposta pela justiça, poderá ser suficiente para que as promessas da administração sejam aceitas como críveis. Por sua vez, em países que tenham um fraco modelo institucional, a construção de reputação é essencial.

Para enfrentar os prováveis problemas advindos de possíveis diferenças entre as relações prometidas e efetivamente praticadas pelas companhias, e com isso colocar em risco a credibilidade do Novo Mercado, a Bolsa de Valores de São Paulo aceita, nesse ambiente de negociação, apenas empresas que concordem em aderir à justiça arbitral, onde eventuais litígios serão debatidos. Para efetivar a adesão, a empresa deve inserir em seu estatuto social uma cláusula padrão, disponível no site da Câmara de Arbitragem do Mercado (Câmara de Arbitragem do Mercado, 2011). Assim, a BM\&F Bovespa visa reduzir, ao menos parcialmente, os eventuais incentivos a desvios, pois poucos subterfúgios legais, que visem descaracterizar o desvio ou postergar sua punição, são possíveis.

A legislação e sua estrutura de compulsão e de garantia de contrato afetam diretamente à complexidade das variáveis que devem ser consideradas em face de novas realidades econômicas. Quando a aplicação da lei e a exigência ao cumprimento de contratos perdem seu caráter impessoal, aumenta drasticamente o grau de incerteza econômica e social. Nesse sentido, a governança corporativa diferenciada pode ter impacto central na dinâmica e na velocidade com a qual uma dada informação é incorporada ao preço de uma ação. Mesmo com normas legais ruins, caso a companhia ou instituição paraestatal, tal como bolsa de valores ou autorregulação, consiga tornar crível a promessa de melhores práticas, deverá ocorrer uma redução nos custos associados ao processamento de novas informações.

Sem que haja reestruturação legal efetiva, a formação de reputação do mercado de governança diferenciada é o principal instrumento pelo qual as empresas podem obter benefícios oriundos da adesão voluntária a um melhor nível de governança. A construção de um mercado capaz de criar os incentivos necessários para tal dinâmica envolve um número considerado de entidades privadas que, voluntariamente, assumem compromissos públicos de bom comportamento. Dentro dessa estrutura, as próprias entidades privadas constroem os mecanismos de punição e recompensa que sustentam o bom funcionamento do mercado.

Um problema fundamental que surge ao se debater Governança Corporativa é a determinação de qual modelo de governança é mais eficiente. Shleifer \& 
Vishny (1997) apontam que a questão principal é garantir proteção legal para um número razoável de investidores, de maneira que o mercado de capitais possa se desenvolver. Em diferentes países, que possuem corporações com bons níveis de governança, a proteção legal é garantida por diferentes combinações de estruturas legais e concentração de propriedade.

Fama \& Jensen (1983), Stulz (1988), Short \& Keasey (1999) apontam que quando o administrador tem uma participação na propriedade suficiente para ter poder de decisão, os mecanismos de governança tendem a perder sua eficiência para proteção dos demais sócios. Jarrel \& Poulsen (1988) encontram evidências de que empresas que possuem duas classes de ações aumentam a capacidade dos administradores em evitar determinados mecanismos de governança, como a possibilidade de aquisição hostil. Para McNabb \& Martin (1988), os acionistas têm considerável ganho de bem-estar quando os fundadores deixam a direção de uma firma.

A principal recompensa esperada por uma empresa que adote um melhor nível de governança é a redução de seu custo de capital, o que deverá ser acompanhado de aumento em seu valor de mercado e maior gama de alternativas de investimentos futuros, o que poderá gerar uma segunda rodada de melhoria em seu valor de mercado e risco.

Para que essa dinâmica seja possível, o mercado como um todo deverá formar uma boa reputação perante o público investidor: sem que essa contraparte acredite na nova estrutura, pouco ou nenhum impacto efetivo ocorrerá e, mesmo que uma firma isolada realmente pratique o melhor nível de governança, os incentivos de mercado serão adversos para a manutenção dessa postura no logo prazo. Porém, com a formação de crença positiva na contraparte investidor, as estruturas de incentivos criadas pelas instituições paraestatais serão legítimas, assim as firmas receberão a devida recompensa pelo bom comportamento. Uma peça fundamental nesse mecanismo é a não intervenção estatal negativa, ou seja, atuação governamental no sentido de proteger empresas que tenham contratualmente aderido a melhores práticas, mas que, por questões conjunturais, optem por romper unilateralmente o contrato. Para sustentabilidade de longo prazo do mercado, ou o governo reforça as estruturas de incentivo e punição privadamente criadas no mercado, ou se abstém de intervir nessas relações.

A construção da reputação está intimamente relacionada ao cumprimento dos dispositivos contratuais, que terminam por limitar de maneira considerável o poder discricionário da administração, ou majoritário. A governança introduz pesos e contrapesos na distribuição do poder interno da empresa, o que ocorre pelo aumento na transparência das informações, nas regras de votação e na composição de conselhos administrativos que limitam o poder dos majoritários e em garantias de determinados direitos aos acionistas minoritários. Um mecanismo subjacente e fundamental é a facilitação de ações civis para reparação de prejuízos causados por má-fé, que no Brasil ocorre através da adesão à Câmara de Arbitragem do Mercado (CAM). 
Ao enquadrar majoritários e administração em um processo de decisão e relacionamento regido por normas claras, a Governança Diferenciada reduziria os custos associados ao processamento de novas informações relevantes, por dois motivos: primeiro, a qualidade das informações prestadas tenderia a ser maior; segundo, dada a maior transparência, as ações da administração e sócios majoritários seriam mais bem alinhadas com o objetivo de maximização do lucro da empresa como um todo, o que levaria a uma redução da busca de benefícios não pecuniários, que seriam obtidos por meio da expropriação do minoritário.

Portanto, um mercado de Governança Diferenciada crível tenderá a ter uma maior velocidade de incorporação de informações do que um mercado com nível de governança ruim. Essa maior velocidade de incorporação de informações está ligada aos custos inerentes à análise das organizações empresarias presentes em cada mercado, sendo as que optam por normas de relacionamento mais claras e menos discricionárias mais fáceis de serem analisadas. É provável, então, que mercados de capitais que possuam empresas com diferentes níveis de governança devam apresentar relações Lead-Lag entre as organizações com menores custos de análise e as com maior custo, ou seja, as de melhor nível de governança e as de pior. É útil notar que, nesse caso, o Lead-Lag não representaria nem oportunidade de arbitragem, dado que não será possível formação de estratégia neutra em risco, nem violação da Hipótese de Eficiência do Mercado (HME), visto que organizações empresariais constituídas com diferentes normas de governança apresentariam funções de incorporação de informação diferentes e não comparáveis.

\section{Metodologia}

Preços de ativos financeiros são naturalmente não estacionários em nível. Para se avaliar a cointegração entre duas séries temporais, é necessário que ambas sejam integradas de mesmo nível.

Caso haja cointegração entre as séries, haverá causalidade no sentido de Granger (1988) em, ao menos, uma direção. Para o caso específico deste estudo, espera-se que haja causalidade de Granger unidirecional do IGC para o IBrX. Ou seja, as alterações no IGC devem ocorrer antes das alterações no IBrX. É útil destacar que a causalidade de Granger tem apenas conotação temporal. Ao se afirmar que o IGC Granger causa o IBrX, não se diz que as alterações do IGC explicam as alterações do IBrX, mas apenas que elas ocorrem antes. É exatamente esse fenômeno que caracterizará a maior velocidade de incorporação de informações do mercado de Governança Diferenciada.

Por sua vez, a cointegração foi testada segundo o método Engle-Granger em dois estágios, conforme utilizado por Brooks et al. (2001), com a inclusão de uma dummy na equação de longo prazo para uma mudança estrutural. Essa abordagem foi proposta por Gregory \& Hansen (1996), que sugeriram o uso de uma dummy na presença de uma quebra estrutural com mudança no intercepto. A relação de longo prazo é apresentada pelas Equações 1 e 2: 


$$
\begin{gathered}
\ln \left(I G C_{t}\right)=\alpha+\beta_{1} \ln \left(I B r X_{t}\right)+\beta_{2} D u+v_{(I G C, t)} \\
\ln \left(I B r X_{t}\right)=\mu+\delta_{1} \ln \left(? I G C_{t}\right)+\delta_{2} D u+v_{(I B r X, t)}
\end{gathered}
$$

onde $\ln \left(I G C_{t}\right)$ é o logaritmo neperiano do Índice de Governança Corporativa; $\ln \left(I B r X_{t}\right)$ é o logaritmo neperiano do Índice Brasil de Ações;

$D u$ é a dummy para uma mudança estrutural no intercepto da relação de cointegração. Igual a 1 para o período 09/11/2009 a 16/12/2009 e zero para os demais; $v_{(I G C, t)}$ é o termo de erro estocástico com média zero e variância constante. É o Mecanismo de Correção de Erro (ECM) da Equação 3, e;

$v_{(I B r X, t)}$ é o termo de erro estocástico com média zero e variância constante. É o Mecanismo de Correção de Erro (ECM) da Equação 4.

Segundo Brooks et al. (2001) e Gregory \& Hansen (1996), se o termo de erro da regressão entre duas variáveis integradas de ordem $1, I(1)$, for integrado de ordem zero, $I(0)$, então pode-se dizer que as variáveis são cointegradas. Para se avaliar a hipótese de estacionaridade dos resíduos, foram realizados dois testes distintos: Aumentado de Dickey-Fuller (ADF) e Phillips-Perron (PP), com ambos sendo realizados sem constante ou intercepto.

Caso haja cointegração entre as séries, então há um VECM tal qual o especificado pelas Equações 2 e 3 . Em virtude dos problemas de heterocedasticidade usualmente encontrados em séries financeiras, o VECM foi estimado com o uso de um modelo GARCH-BEKK, o que segue Pati \& Rajib (2010). As Equações 3 e 4 apresentam o VECM:

$$
\begin{aligned}
\Delta I G C_{t} & =\vartheta+\sum_{i=1}^{p-1} \alpha_{I G C, i} \Delta I G C_{t-i}+\sum_{i=1}^{p-1} b_{I G C, i} \Delta I B r X_{t-i} \\
& +\alpha_{I G C} E C M_{I G C, t-1}+\epsilon_{I G C, t} \\
\Delta I B r X_{t} & =\varphi+\sum_{i=1}^{p-1} \alpha_{I B r X, i} \Delta I C_{t-i}+\sum_{i=1}^{p-1} b_{I B r X, i} \Delta I B r X_{t-i} \\
& +\alpha_{I B r X} E C M_{I B r X, t-1}+\epsilon_{I B r X, t}
\end{aligned}
$$

onde $\alpha_{(I G C, i)}, \alpha_{(I B r X, i)}, b_{(I G C, i)}$ e $b_{(I B r X, i)}$ são coeficientes de curto prazo; $E C M_{(}(i, t-1)$ é o mecanismo de Correção de Erro, igual a $\left.v_{(} i, t\right)$ das Equações 1 e 2 ;

$\epsilon_{(I G C, t)}$ e $\epsilon_{(I B r X, t)}$ são resíduos, com $\sim t(0, H t) ;$ 
$\Delta I G C_{t}: R_{i g c, t}=\ln \left(I G C_{t}\right)-\ln \left(I G C_{(t-1)}\right), \mathrm{e} ;$

$\Delta I B r X_{t}: R_{i g c, t}=\ln \left(I B r X_{t}\right)-\ln \left(I B r X_{t-1}\right)$.

Para que o IGC Granger cause o IBrX unidirecionalmente, é necessário que:

i. algum dos coeficientes $\alpha_{I B r X, i}, i=1,2, \ldots, p-1$ seja diferente de zero; e/ou,

ii o coeficiente de correção de erro, $\alpha_{I B r X}$, seja estatisticamente significativo.

Já a causalidade de Granger unidirecional do IBrX para o IGC ocorrerá se:

iii. algum dos coeficientes $b_{I G C, i}, i=1,2, \ldots, p-1$ seja diferente de zero; e/ou,

iv o coeficiente de correção de erro, $\alpha_{I G C}$, seja estatisticamente significativo.

Séries financeiras apresentam uma característica chamada agrupamento, onde "momentos tranquilos de pequenos retornos são intercalados com períodos voláteis de grandes retornos. ... esse fenômeno é [conhecido como] heterocedasticidade condicional auto-regressiva" (Alexander, 2005, p. 67), e resulta em forte autocorrelação no quadrado da primeira diferença de séries de preço.

Ao se trabalhar com modelos GARCH, admite-se como hipótese retornos gerados por processo estocástico com volatilidade variável no tempo; em um modelo GARCH, trabalha-se com hipóteses específicas e mais detalhadas sobre o comportamento temporal da variância do termo de erro, que é modelada através de uma equação específica chamada Equação da Variância Condicional.

Segundo Teräsvirta (1996), modelos GARCH normais falham na captura do excesso de curtose presente em dados de alta frequência. Para esses casos, Bollerslev (1987) e Engle \& González-Riviera (1991) propõem que se admita uma distribuição leptocúrtica no processo do erro da equação da média condicional, o que pode ser obtido com o uso de um modelo $t \mathrm{GARCH}$, que assume uma distribuição $t$ do erro.

Adicionalmente, modelos GARCH multivariados exigem a estimação de um grande número de parâmetros, o que pode resultar em graves problemas computacionais. Por isso optou-se pelo uso do GARCH-BEKK (Engle \& Kroner, 1995), que reduz o número de parâmetros estimados e garante que a matriz da variância condicional seja positiva definida. Para melhor performance, o modelo adotado é um BEKK diagonal, cujo desempenho é superior ao BEKK escalar (Engle, 2000).

Nesse sentido, para a estimativa do modelo VECM-GARCH-BEKK, assumiuse uma distribuição condicional $t$ de Student como função densidade do termo de erro e uma variância condicional, conforme especificada pela Equação 6:

$$
\begin{aligned}
H_{t} & =A^{\prime} A+B^{\prime} H_{t-1} B+C^{\prime} \epsilon_{t-1} \epsilon_{t-1}^{\prime} C+T 1^{\prime} u_{1, t-1} u_{1, t-1}^{\prime} T 1 \\
& +T 2^{\prime} u_{2, t-1} u_{2, t-1}^{\prime} T 2+E^{\prime}\left(z_{t-1}\right)^{2} E
\end{aligned}
$$


onde $H_{t}$ é a variância condicional do termo de erro;

$A$ é a matriz triangular $2 \times 2$ de coeficientes;

$B$ e $C$ são matrizes diagonais de coeficientes;

T1 e T2 são matrizes com parâmetros de efeito spillover;

$u_{1, t-1}$ é a matriz com o quadrado do termo de erro defasado. Efeito spillover do IBrX para o IGC;

$u_{2, t-1}$ é a matriz com o quadrado do termo de erro defasado. Efeito spillover do IGC para o IBrX;

$z_{t-1}$ é a defasagem da base (ECM), e;

$E$ é o vetor $1 \mathrm{x} 2$ de parâmetros estimados para a defasagem da base.

O modelo de variância condicional adotado na Equação 6 assume que a variância condicional é função de notícias antigas (valores defasados), notícias novas (termos de erro defasados), parâmetros de spillover (transbordamento) e do quadrado do parâmetro base, enquanto a covariância condicional é função da covariância defasada e do produto cursado dos termos de erro originais. Essa abordagem segue Pati \& Rajib (2010).

O modelo GARCH-BEKK foi estimado por máxima verossimilhança. A Equação 7 apresenta a formulação geral da função de verossimilhança utilizada.

$L\left(\epsilon_{t}, H_{t}\right)=\frac{G\left[\frac{(2+v)}{2}\right]}{G\left(\frac{v}{2}\right)[p(v-2)]}\left|H_{t}\right|^{-\frac{1}{2}}\left[1+\frac{1}{v-2} \epsilon_{t}^{\prime}, H_{t}^{-1}\right]^{-[\operatorname{frac}(2+v) 2]}$, para $v>2$

onde $G($.$) é uma função gama, e;$

$v$ é o número de graus de liberdade.

\section{Dados e ECM}

Para a estimativa do efeito Lead-Lag foram utilizados dados na frequência de 15 minutos dos Índices de Governança Corporativa Diferenciada (IGC) e do Índice Brasil de Ações (IBrX 100), com um total de 5.582 observações de preços e 5.581 observações de retornos. A série de dados tem início em maio de 2009 e término em fevereiro de 2010. Ambos os índices possuem empresas em comum, o que torna mais difícil a constatação de eventuais efeitos Lead-Lag.

O IBrX é uma carteira formada pelas 100 principais empresas com maior índice de negociabilidade nos pregões da BM\&F Bovespa, enquanto o IGC contém apenas empresas listadas nos mercados de Governança Diferenciada, cujos pesos no índice são ponderados pelo nível de governança da companhia ${ }^{1}$ (BM\&F Bovespa, 2011).

Durante o período analisado, o IGC possuía 174 ativos, dos quais 98 não fazem parte do IBrX, o que equivale a 17,08\% do valor total do IGC. Por sua vez, o IBrX

\footnotetext{
${ }^{1}$ A metodologia completa de construção dos índices pode ser encontrada em www. bmfbovespa. com.br/indices/BuscarIndices . aspx?idioma=pt-br
} 
era composto por 100 ações, das quais 23 não constavam na carteira do IGC e equivaliam a $29,67 \%$ do valor total do $\mathrm{IBrX}$.

Quanto às ações em comum, elas possuem uma participação média 11,47\% maior no IGC do que no IBrX, com um desvio-padrão associado a essa média de $82,88 \%$.

A impossibilidade de se incorporar variáveis de controle para diferentes características entre o IGC e IBrX que não estejam ligadas à Governança Corporativa é uma importante restrição metodológica e implica na necessidade de se observar com cuidado as conclusões.

Apesar da alta correlação gerada pela intercessão entre os conjuntos de ativos dos dois índices, a diferença marginal entre eles é assumida como suficiente para que o processo gerador de dados das duas carteiras produza velocidades de convergência ao equilíbrio e influências recíprocas diferentes, principalmente em dados de alta frequência.

Dado as Equações1 e 2, é tautológica a conclusão de que $\epsilon_{I G C, t}$ é o inverso de $\vartheta_{I B r X, t}$. Para a análise dos resultados da convergência ao equilíbrio no contexto da relação de Lead-Lag, dois fatores são essenciais: o sinal e a significância estatística do parâmetro estimado para o ECM.

Um parâmetro estimado para o ECM, na Equação 3 negativo e significativo, indica que, se a diferença entre o IBrX e o IGC for positiva em um período, o IBrX cairá no próximo período para restaurar o equilíbrio; se a diferença for negativa, o IBrX subirá (Brooks et al., 2001). Por sua vez, um sinal positivo e significante indicará que o IBrX não converge para a posição de equilíbrio indicada pelo IGC. Para a Equação 2, a interpretação é, com a devida substituição de IBrX por IGC e vice versa, a mesma.

Com a conclusão de que um ECM será o inverso do outro, surge que, em uma das Equações 2 ou 3, o parâmetro estimado deverá ser positivo, enquanto na outra negativo. Porém, dado que a variância dos índices é diferente, o fato do ECM ser estatisticamente significante em uma das equações não implica que será na outra.

\section{Análise e Resultados}

A hipótese testada no presente trabalho é de que um melhor nível de Governança Corporativa reduz os custos associados à incorporação de novas informações aos preços dos ativos da empresas. Dessa forma, a velocidade de incorporação de informações apresentada por ativos de empresas com bons níveis de governança deve ser superior a de companhias com estruturas de governanças mais fracas. Caso a hipótese seja válida, o mercado de Governança Diferenciada deverá liderar o mercado tradicional. A presente seção lista e comenta os resultados encontrados pelo trabalho. 


\subsection{Análise preliminar dos dados}

A Tabela 1 apresenta as estatísticas da série de retornos na frequência de 15 minutos do Índice de Governança Corporativa Diferenciada (IGC) e do Índice Brasil de Ações (IBrX). Para o período analisado, o retorno médio do IGC foi cerca de $33 \%$ maior que o do IBrX, com desvio padrão pouco menor. Ambas as séries apresentam assimetria negativa e excesso de curtose, sendo a série de retornos do IGC mais assimétrica. O teste Jarque-Bera foi altamente significativo, o que implica na rejeição de hipótese nula de normalidade na distribuição dos retornos.

Tabela 1

Sumários das estatísticas

\begin{tabular}{lcc}
\hline & $\Delta I G C_{t}$ & $\Delta I B r X_{t}$ \\
\hline Média & 0,00006 & 0,000045 \\
Mediana & 0,00009 & 0,000067 \\
Máximo & 0.0216 & 0.0201 \\
Mínimo & -0.0267 & -0.0238 \\
Desvio Padrão & 0.0026 & 0.0027 \\
Assimetria & -0.2101 & -0.0935 \\
Curtose & 17.0476 & 13.2260 \\
\hline \multicolumn{2}{c}{} \\
\hline Jarque-Bera & 45929,60 & 24325,03 \\
Valor-p & 0.00 & 0.00 \\
\hline & & \\
Observações & 5581 & 5581 \\
\hline
\end{tabular}

A Tabela 2 apresenta o cálculo do coeficiente de correlação de Pearson entre as séries. Como pode ser visto, há uma forte correlação entre os retornos dos dois índices de mercado.

Tabela 2

Correlação

\begin{tabular}{lcc}
\hline & $\Delta I G C_{t}$ & $\Delta I B r X_{t}$ \\
\hline$\Delta I G C_{t}$ & 1.000 & 0.973 \\
$\Delta I B r X_{t}$ & 0.973 & 1.000 \\
\hline
\end{tabular}

Os resultados dos testes, em nível e em primeira diferença, ADF e PP de raiz unitária são apresentados na Tabela 3. Os dois testes foram calculados sem tendência ou intercepto e possuem resultados convergentes. Indicam que, para um nível de significância de 5\%, as séries não são estacionárias em nível, mas são estacionárias em primeira diferença, sendo por tanto $I(1)$. 
Tabela 3

Testes de raiz unitária

\begin{tabular}{lcc}
\hline Variáveis & ADF & PP \\
\hline Nível $(\log )$ & & \\
IGCt & 1,715 & 1,571 \\
IBrXt & 1,212 & 1,139 \\
\hline & & \\
\hline Primeira diferença & & \\
$\Delta I G C_{t}$ & $-73,060^{*}$ & $-73,335^{*}$ \\
$\Delta I B r X t$ & $-75,145^{*}$ & $-75,280^{*}$ \\
\hline
\end{tabular}

\subsection{Estimativa do modelo}

Dado que ambas as séries são integradas de mesma ordem, então a relação de longo prazo entre elas pode ser calculada com base nas Equações 1 e 2 (Brooks et al., 2001). Os resultados dos parâmetros estimados são apresentados na Tabela 4.

Tabela 4

Relação de longo prazo

\begin{tabular}{ccc}
\hline \multicolumn{3}{l}{$\ln \left(I B r X_{t}\right)=\mu+\delta_{1} \ln \left(I G C_{t}\right)+\delta_{2} D u+\vartheta_{I B r X, t}$} \\
\hline Coeficiente & Erro Padrão & Estatística-t \\
\hline 2,8672 & 0,0124 & 230,9311 \\
0,8056 & 0,0014 & 561,2514 \\
0,0264 & 0,0005 & 49,259 \\
\hline \multicolumn{3}{c}{} \\
\hline \multicolumn{3}{c}{$\ln \left(I G C_{t}\right)=\alpha+\beta_{1} \ln \left(I B r X_{t}\right)+\beta_{2} D u+\vartheta_{I G C, t}$} \\
\hline Coeficiente & Erro Padrão & Estatística-t \\
\hline$-3,3466$ & 0,0214 & $-156,573$ \\
1,2197 & 0,0022 & 561,2514 \\
$-0,0298$ & 0,0007 & $-43,721$ \\
\hline
\end{tabular}

O parâmetro calculado para a variável dummy de mudança estrutural é significativo em ambas as equação. Sua introdução no modelo seguiu Gregory \& Hansen (1996) e indica uma mudança temporária no intercepto da relação de longo prazo.

Os resíduos $\vartheta_{I B r X, t}$ e $\vartheta_{I G C, t}$ são os parâmetros de cointegração. Pelo método Engle-Granger em dois estágios, se os resíduos da relação de longo prazo de duas variáveis $I(1)$ forem estacionários, então as séries cointegram. Para se avaliar a estacionaridade dos resíduos, testes ADF e PP, sem tendência ou intercepto, foram realizados; os resultados são apresentados na Tabela 5. 
Tabela 5

Testes de raiz unitária

\begin{tabular}{lcc}
\hline Variáveis & ADF & PP \\
\hline$\vartheta_{I G C, t}$ & $-2,517^{*}$ & $-2,488^{*}$ \\
$\vartheta_{I B r X, t}$ & $-2,655^{*}$ & $-2,629^{*}$ \\
\hline
\end{tabular}

Ambos os testes indicam rejeição da hipótese nula de existência de raiz unitária ao nível de significância de 5\%. Pelo método de Engle-Granger em dois estágios, esse resultado indica que as séries cointegram.

Segundo o Teorema da Representação de Granger (Maddala \& Kim, 1998), se duas séries cointegram, então existe um VECM para essa relação de cointegração. Neste estudo, o VECM calculado foi formalizado nas Equações 3 e 4. Conforme apresentado na metodologia, séries financeiras apresentam problema de heterocedasticidade, por isso optou-se pelo uso de um modelo GARCH-BEKK, conforme especificado por Pati \& Rajib (2010). Para determinação do número ótimo de lags foi utilizado o critério de Schwartz $(-21,827)$, que indicou uma defasagem ótima de apenas um lag. Segundo, Brooks (2008), o critério de Schwartz é o mais recomendável para comparação entre diferentes modelos com grande número de observações.

Os resultados calculados para o VECM são apresentados na Tabela 6; foram necessárias 25 iterações para estimação do modelo.

O Mecanismo de Correção de Erro (ECM) fornece a velocidade com a qual a séria converge para a relação de equilíbrio de longo prazo. Para o VECM do Índice de Governança Corporativa (IGC), o coeficiente estimado para o $E C M_{I G C, t-1}$ é positivo, mas estatisticamente não significativo; isso implica que o IGC não responde ao desvio da relação de equilíbrio do período anterior. Por sua vez, o parâmetro calculado para o $E C M_{I B r X, t-1}$ no VECM do Índice Brasil de Ações (IBrX) é negativo e, ao nível de 5\%, significante, o que, segundo Brooks et al. (2001), implica que caso a diferença entre o IGC e o IBrX seja positiva em um período, o IBrX cairá no próximo período para restaurar o equilíbrio; se a diferença for negativa, o IBrX subirá.

Nenhum dos parâmetros calculados para defasagens do IGC ou IBrX é significativo no VECM do IGC. Para o VECM do IBrX, todos os parâmetros calculados para a defasagem são significativos. Isso implica que, enquanto toda informação gerada no período anterior já foi incorporada no IGC, ainda não foi totalmente incorporada no IBrX, o que indica uma menor velocidade de incorporação de informações do último. 
Tabela 6

Modelo VECM

\begin{tabular}{|c|c|c|c|}
\hline \multicolumn{4}{|c|}{$\begin{array}{c}\Delta I G C_{t}=\vartheta+\sum_{i=1}^{p-1} \alpha_{I G C, i} \Delta I G C_{t-i}+\sum_{i=1}^{p-1} b_{I G C, i} \Delta I B r X_{t-i} \\
+\alpha_{I G C} E C M_{I G C, t-1}+\epsilon_{I G C, t}\end{array}$} \\
\hline Coeficiente & Erro Padrão & Estatística-t & Valor $\mathrm{p}$ \\
\hline 0.0001 & 0.0000 & 41.849 & 0.0000 \\
\hline 0.0397 & 0.0408 & 0.9725 & 0.3308 \\
\hline 0.0019 & 0.0013 & 15.542 & 0.1201 \\
\hline-0.0145 & 0.0378 & -0.3844 & 0.7006 \\
\hline \multicolumn{4}{|c|}{$\begin{array}{c}I B r X_{t}= \\
\varphi+\sum_{\left.(i=1)^{(} p-1\right) \alpha_{I B r X, i} \Delta I G C_{t-i}+\sum_{i=1}^{p-1} b_{I B r X, i}} \\
\Delta I B r X_{t-i}+\alpha_{I B r X} E C M_{I B r X, t-1}+\epsilon_{I B r X, t}\end{array}$} \\
\hline Coeficiente & Erro Padrão & Estatísitca-t & Valor $\mathrm{p}$ \\
\hline 0.0001 & 0.0000 & 35.692 & 0.0004 \\
\hline 0.1099 & 0.0436 & 25.180 & 0.0118 \\
\hline-0.0037 & 0.0017 & -21.613 & 0.0307 \\
\hline-0.1077 & 0.0416 & -25.877 & 0.0097 \\
\hline
\end{tabular}

Os resultados estimados para a equação da variância são apresentados na Tabela 7. Todas as variáveis foram estaticamente significativas. A evidência empírica aponta para existência de efeito bidirecional de transbordamento da volatilidade entre o IGC e IBrX, assim como há indício de dependência temporal na volatilidade, sendo a volatilidade presente explicada parcialmente pela volatilidade passada.

Tabela 7

Equação da variância

\begin{tabular}{|c|c|c|c|c|}
\hline \multicolumn{5}{|c|}{$\begin{aligned} G A R C H & =M+A 1 * R E S I D(-1) * R E S I D(-1)^{\prime} \\
& * A 1+B 1 * G A R C H(-1) * B 1\end{aligned}$} \\
\hline & Coeficiente & Erro Padrão & Estatística-z & Valor $\mathrm{p}$ \\
\hline $\mathrm{M}(1,1)$ & $1.48 \mathrm{E}-06$ & $1.39 \mathrm{E}-07$ & 10.6864 & 0.0000 \\
\hline $\mathrm{M}(1,2)$ & $1.47 \mathrm{E}-06$ & $1.46 \mathrm{E}-07$ & 10.1066 & 0.0000 \\
\hline $\mathrm{M}(2,2)$ & $1.53 \mathrm{E}-06$ & $1.66 \mathrm{E}-07$ & 9.20909 & 0.0000 \\
\hline $\mathrm{A} 1(1,1)$ & 0.377187 & 0.020364 & 18.5222 & 0.0000 \\
\hline $\mathrm{A} 1(2,2)$ & 0.326039 & 0.018487 & 17.6363 & 0.0000 \\
\hline $\mathrm{B} 1(1,1)$ & 0.793383 & 0.015621 & 50.7902 & 0.0000 \\
\hline $\mathrm{B} 1(2,2)$ & 0.836144 & 0.014763 & 56.6374 & 0.0000 \\
\hline
\end{tabular}

A Tabela 8 apresenta os resultados calculados para o teste de causalidade de Granger. A hipótese nula de que o IBrX não Granger causa o IGC não pode ser rejeitada ao nível de significância de 5\%. Entretanto, a hipótese nula de que o IGC não Granger causa o IBrX é rejeitada aos mesmos 5\% de significância. Portanto, pode-se concluir que há causalidade de Granger unidirecional do IGC para o IBrX.

O conjunto dos resultados encontrados apontam que variações no IGC precedem as variações do IBrX e que o IBrX converge para a relação de equilíbrio determinada pelo IGC. 
Tabela 8

Causalidade de Granger

\begin{tabular}{lcc}
\hline Hipótese nula & Estatística $\mathrm{F}$ & Valor $\mathrm{p}$ \\
\hline$\Delta I B r X$ não Granger causa $\Delta I G C$ & 0.00117 & 0.973 \\
$\Delta I G C$ não Granger causa $\Delta I B r X$ & 6.83719 & 0.009 \\
\hline
\end{tabular}

\section{Conclusão}

O presente estudo analisou a relação de Lead Lag entre o Índice Brasil de Ações (IBrX) e o Índice de Governança Corporativa Diferenciada (IGC). A hipótese básica testada foi de que melhores práticas de governança facilitam a incorporação de novas informações ao preço dos ativos. Dessa forma, o mercado de Governança Diferenciada, IGC, deveria liderar o mercado tradicional, IBrX.

As evidências encontradas não permitem a rejeição da hipótese proposta e indicam que o mercado de governança parece liderar o mercado tradicional. Foi encontrada causalidade unidirecional de Granger do IGC para o IBrX, assim como indícios, apontados pelo sinal e significância estatística do Mecanismo de Correção de Erros (ECM), de que o mercado de governança diferenciada encontra seu preço de equilíbrio de longo prazo mais rapidamente que o mercado tradicional, e que o último converge para a relação de equilíbrio apontada pelo primeiro.

É importante ressaltar que o efeito Lead-Lag observado pode também estar ligado a outras diferenças sistemáticas entre as empresas dos dois grupos, por exemplo, maior cobertura por analistas das empresas do IGC independentemente das práticas de GC ou simplesmente porque as empresas do IGC são menos complexas em função da natureza de sua atividade. Assim, deve-se considerar que a dificuldade de se controlar os efeitos de outras variáveis que podem contribuir para o fenômeno deve ser vista como importante limitador das conclusões aqui apresentadas.

Com relação às implicações dos resultados obtidos para a Hipótese de Mercados Eficientes, não há indícios que apontem para sua violação. É possível que a maior velocidade de incorporação de informações encontrada para o mercado de governança mais elevada seja resultado dos menores custos associados à incorporação das novas informações ao preço. Uma possível explicação para isso seria que, ao adotar regras mais claras, as empresas com melhores governanças possibilitariam um acompanhamento mais eficiente pelos analistas quanto às implicações de novos fatos relevantes. Assim, o efeito Lead-Lag poderia estar associado à existência de custos de processamento de informações diferenciados para os analistas das diferentes empresas. Não é possível, entretanto, descartar a hipótese de que o efeito Lead-Lag em questão possa ser explicado por outros fatores.

Sugere-se o aprofundamento dos estudos sobre a relação entre Governança Corporativa e velocidade de incorporação de informações como agenda de pesquisa para trabalhos futuros. Um possível desdobramento é a análise de quais características de governança estão mais relacionadas com o aumento da velocidade de incorporação de informações. 


\section{Referências}

Alexander, Carol. 2005. Modelos de Mercado. São Paulo: BM\&F e Editora Saraiva.

Bhojraj, Sanjeev, \& Sengupta, Partha. 2003. Effects of Corporate Governance on Bond Ratings and Yields: The Role of Institutional Investors and Outside Directors. Journal of Business, 76, 455-475.

Black, Bernard. 2001. The Corporate Governance Behavior and Market Value of Russian Firms. Emerging Markets Review, 2, 89-108.

Black, Bernard S., Carvalho, Antônio G. de, \& Gorga, Erica. 2009. The Corporate Governance of Privately Controlled Brazilian Firms. Revista Brasileira de Finanças, 7, 385-428.

Bollerslev, Tim. 1987. A Conditional Heteroskedasticity Time Series Model for Security Prices and Rates of Return Data. Review of Economics and Statistics, 69, 542-547.

Botosan, Chistine A., Plumlee, Marlene A., \& Xie, Yuan. 2004. The Role of Information Precision in Determining the Cost of Equity Capital. Review of Accounting Studies, 9, 233-259.

Brenann, Michael J., Jegadeesh, Narasimhan, \& Swaminathan, Bhaskaran. 1993. Investment Analysis and the Adjustment of Stock Prices to Common Information. The Review of Financial Studies, 6, 799-824.

Brooks, Chris. 2008. Introductory Econometrics for Finance. Second edn. Cambridge: Cambridge Press.

Brooks, Chris, Rew, Alistair G., \& Ritson, Stuart. 2001. A Trading Strategy Based on the Lead-Lag Relationship Between the Spot Index and Future Contract or the FTSE. International Journal of Forecasting, 17, 31-44.

Bushman, Robert, Chen, Qi, Engel, Ellen, \& Smith, Abbie. 2004. Financial Accounting Informational, Organization Complexity and Corporate Governance Systems. Journal of Accounting \& Economics, 37, 167-201.

Byrne, Alistair, \& Brooks, Mike. 2008. Behavioral Finance: Theories and Evidence. The Research Foundation of CFA Institute Literature Review, 3, 1-26.

Carvalho, Antônio G. de. 2002. Governança Corporativa No Brasil Em Perspectiva. Revista de Administração da Universidade de São Paulo, 37, 19-33.

Chan, Wesley S. 2003. Stock Price Reaction to News and No-News: Drift and Reversal After Headlines. Journal of Financial Economics, 70, 223-260. 
Cutler, David M., Poterba, James M., \& Summers, Lawrence H. 1989. What Moves Stock Prices? Journal of Portfolio Management, 15, 4-12.

Easley, David, \& O'Hara, Maureen. 2004. Information and the Cost of Capital. The Journal of Finance, 59, 1553-1583.

Engle, Robert F. 2000. Dynamic Conditional Correlation: A Simple Class of Multivariate GARCH Models. Working Paper, disponível em: http://weber. ucsd. edu/ mbacci/engle/cv.html.

Engle, Robert F., \& González-Riviera, Gloria. 1991. Semi-Parametric GARCH Models. Journal of Business and Economic Statistics, 9, 345-359.

Engle, Robert F., \& Kroner, Kenneth F. 1995. Multivariate Simultaneous Generalized Arch. Econometric Theory, 1, 122-150.

Fama, Eugene F. 1991. Efficient Capital Markets: II. The Journal of Finance, 46, $1575-1617$.

Fama, Eugene F., \& Jensen, Michael. 1983. Agency Problems and Residual Claims. Journal of Law and Economics, 26, 327-349.

Goergen, Marc, \& Renneboog, Luc. 2008. Contractual Corporate Governance. Journal of Corporate Finance, 14, 166-182.

Gompers, Paul, Ishii, Joy L., \& Metrick, Andrew. 2003. Corporate Governance and Equity Price. The Quarterly Journal of Economics, 118, 107-155.

Granger, Clive W. J. 1988. Some Recent Developments in a Concept of Causality. Journal of Econometrics, 39, 199-211.

Gregory, Allan W., \& Hansen, Brunce E. 1996. Residual-Based Test for Cointegration in Models With Regime Shift. Journal of Econometrics, 70, 99-126.

Jarrel, Gregg, \& Poulsen, Annette B. 1988. Dual-Class Recapitalizations as Antitakeover Mechanisms: The Recent Evidence. Journal of Financial Economics, 20, 129-152.

Jensen, Michael C. 1978. Some Anomalous Evidence Regarding Market Efficiency. Journal of Financial Economics, 6, 95-101.

Jensen, Michael C., \& Meckling, William H. 1976. Theory of the Firm: Managerial Behavior, Agency Cost and Ownership Structure. Journal of Financial Economics, 3, 305-360.

Joh, Sung W. 2003. Corporate Governance and Firm Profitability: Evidence from Korea Before the Economic Crisis. The Journal of Financial Economics, 68, 287-322. 
LaPorta, Rafael, Lopez-de Silandes, Florencio L., Shleifer, Andrei, \& Vishny, Robert W. 1998. Law and Finance. Journal of Political Economy, 106, 1113 1155 .

LaPorta, Rafael, Lopez-de Silandes, Florencio L., Shleifer, Andrei, \& Vishny, Robert W. 2000. Investor Protection and Corporate Governance. Journal of Financial Economics, 58, 3-27.

Lucas, Robert E. Jr. 1978. Asset Prices in an Exchange Economy. Econometrica, 46, 1429-1445.

Lucas, Robert E. Jr. 1986. Behavior and Economics Theory. The Journal of Business, 59, S401-S426.

Maddala, Gangadharrao S., \& Kim, In-Moon. 1998. Unit Roots, Cointegration, and Structural Change. Cambridge: Cambridge Press.

Madhavan, Ananth. 2000. Market Microstructure: A Survey. Journal of Financial Markets, 3, 205-258.

McNabb, Mark M., \& Martin, John D. 1988. Managerial Entrenchment and Effectiveness of Internal Governance Mechanisms. Working Paper, Virginia Tech University and University of Texas at Austin.

Oliveira, Gustavo R., \& Medeiros, Otávio R. 2009. Testando a Existência de Efeitos Lead-Lag Entre Os Mercados Acionários Norte-Americano e Brasileiro. Brazilian Business Review, 6, 1-21.

Pati, Pratap C., \& Rajib, Prabina. 2010. Intraday Return Dynamics and Volatility Spillovers Between NSE S\&P CNX Nifty Stock Index and Index Futures. Applied Economics Letters, 1-8.

Plumlee, Marlene A. 2003. The Effect of Information Complexity on Analysts' Use of That Information. The Accounting Review, 78, 275-296.

Poshakwale, Sunil S., \& Theobald, Michael. 2004. Market Capitalisation, CrossCorrelations, the Lead-Lag Structure and Microstructure Effects in the Indian Stock Market. Journal of International Financial Markets, Institutions and Money, 14, 385-400.

Roe, Mark. J. 2005. The Institutions of Corporate Governance. Pages 371-399 of: Ménard, C., \& Shirlet, M. M. (eds), Handbook of New Institutional Economics. Berlin: Springer.

Romano, Roberta. 1996. Corporate Law and Corporate Governance. Industrial and Corporate Change, 5, 277-339. 
Shleifer, Andrei, \& Vishny, Robert W. 1997. A Survey of Corporate Governance. The Journal of Finance, 52, 737-783.

Short, Helen, \& Keasey, Kevin. 1999. Managerial Ownership and the Performance of Firms: Evidences from UK. Journal of Corporate Finance, 5, 79-101.

Silva, André L. C. Da. 2004. Governança Corporativa, Valor, Alavancagem e Políticas de Dividendos Das Empresas Brasileiras. Revista de Administração da Universidade de São Paulo, 39, 348-361.

Silva, André L. C. Da, \& Leal, Ricardo P. C. 2005. Corporate Governance Index, Firm Valuation and Performance in Brazil. Revista Brasileira de Finanças, 3, $1-18$.

Silveira, Alexandre D. M., Barros, Lucas A. B. de C., \& Famá, Rubens. 2006. Atributos Corporativos, Qualidade Da Governança Corporativa e Valor Das Companhias Abertas No Brasil. Revista Brasileira de Finanças, 4, 1-30.

Silveira, Alexandre D. M., Perobelli, Fernanda F. C., \& Barros, Lucas A. B de C. 2008. Governança Corporativa e Os Determinantes Da Estrutura de Capital: Evidências Empíricas No Brasil. Revista de Administração Contemporânea [online], 12, 763-788.

Srour, Gabriel. 2005. Práticas Diferenciadas de Governança Corporativa: Um Estudo Sobre a Conduta e Performance Das Firmas Brasileiras. Revista Brasileira de Economia, 59, 635-674.

Stulz, René M. 1988. Managerial Control of Voting Rights: Financing Policies and the Market for Corporate Control. Journal of Financial Economics, 20, 25-54.

Sunders, Shyam. 1992. Market for Information: Experimental Evidence. Econometrica, 60, 667-695.

Teräsvirta, Timo. 1996. Two Stylized Facts and the $\operatorname{GARCH}(1,1)$ Modal. Stocholm School of Economics Working Paper Series in Economics and Finance, 96.

Williamson, Oliver E. 1983. Credible Commitments: Using Hostages to Support Exchange. The American Economic Review, 73, 519-540.

Williamson, Oliver E. 1984. Corporate Governance. Yale Law Journal, 93, 1197 1230.

Williamson, Oliver E. 1988. Corporate Finance and Corporate Governance. Governance. The Journal of Finance, 43, 567-591. 